\title{
Neutrophil elastase inhibition improves intestinal mucosal damage and gut microbiota in a mouse model of 5-fluorouracil-induced intestinal mucositis
}

\author{
Kung-Ju Chen ${ }^{1}$, Yu-Li Chen ${ }^{2}$, Shir-Hwa Ueng ${ }^{3}$, Liang-Mou Kuo ${ }^{4}$, and Pei-Wen Hsieh ${ }^{1}$ \\ ${ }^{1}$ Chang Gung University Graduate Institute of Biomedical Sciences \\ ${ }^{2}$ Chang Gung University College of Medicine \\ ${ }^{3}$ Chang Gung Memorial Hospital \\ ${ }^{4}$ Chang Gung Memorial Hospital Department of Surgery
}

May 5, 2020

\begin{abstract}
Background and Purpose: 5-Fluorouracil (5-FU)-based chemotherapy is the first-line chemotherapeutic agent for colorectal cancer. However, 5-FU-induced intestinal mucositis (FUIIM) is a common adverse effect that severely impairs drug tolerance and results in poor patient health. Experimental Approach: Neutrophil elastase (NE) overexpression contributes to FUIIM via abnormal inflammatory responses, microbiota imbalance, and tissue damage. Therefore, restoring NE homeostasis could prevent or improve FUIIM. Key Results: This study shows that treatment with the specific NE inhibitor MPH966 (7.5 mg/kg; p.o.) significantly reversed 5-FU-induced losses in body weight; reversed villus atrophy; significantly suppressed myeloperoxidase, $\mathrm{NE}$, and proteinase 3 activity; and reduced pro-inflammatory cytokine levels in a mouse model of FUIIM. In addition, MPH966 prevented 5-FU-induced intestinal barrier dysfunction, as was indicated by modulated expression of the tight junction proteins zonula occludin-1 and occludin. MPH966 also reversed 5-FU-induced changes in gut microbiota diversity and abundances, specifically the Firmicutes-to-Bacteroidetes ratio; Muribaculaceae, Ruminococcaceae, and Eggerthellaceae abundances at the family level; and Candidatus Arthromitus abundance at the genus level. Conclusion and Implications: These data indicate that NE inhibitors are a potential treatment candidate to alleviate FUIIM by regulating abnormal inflammatory responses, intestinal barrier dysfunction, and gut microbiota imbalance.
\end{abstract}

\section{Hosted file}

Main Text.pdf available at https://authorea.com/users/307244/articles/438205-neutrophilelastase-inhibition-improves-intestinal-mucosal-damage-and-gut-microbiota-in-a-mousemodel-of-5-fluorouracil-induced-intestinal-mucositis 
A

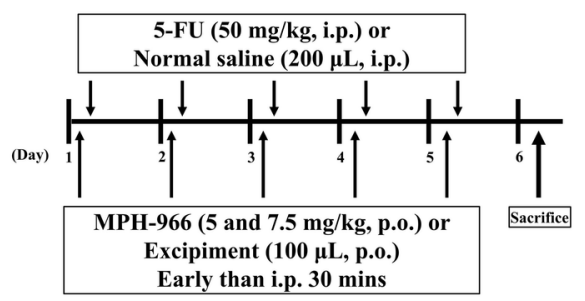

C
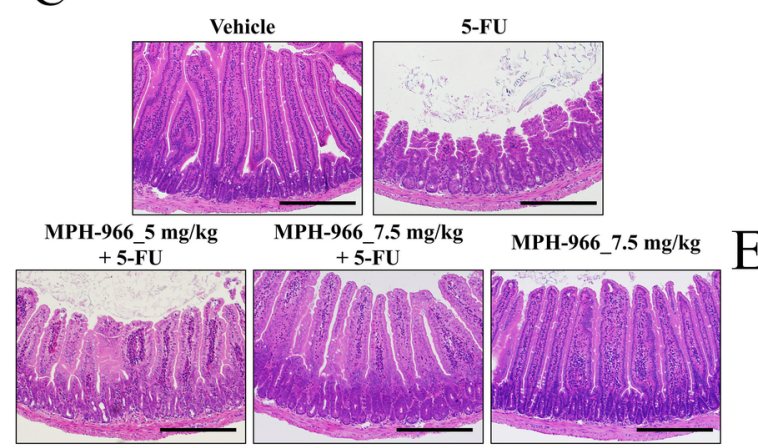

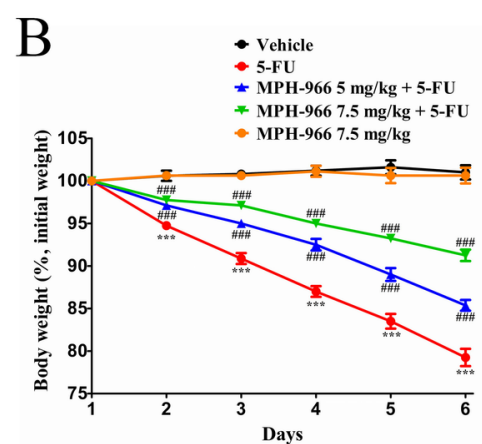

D

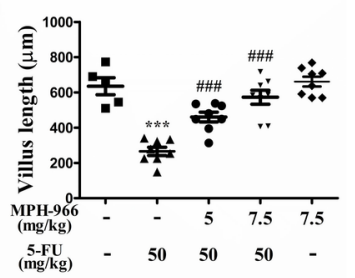

E

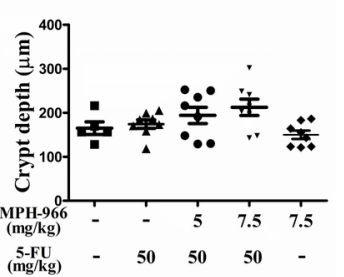

C
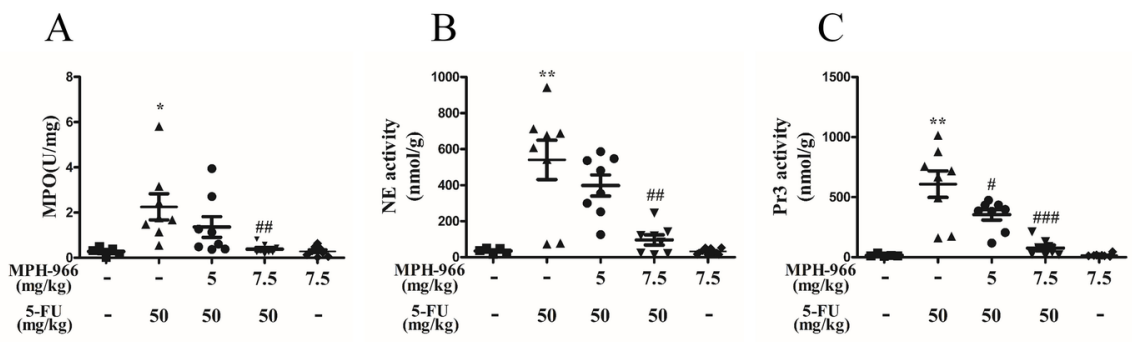

D

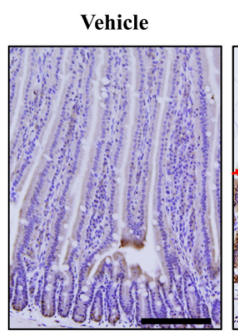

5-FU
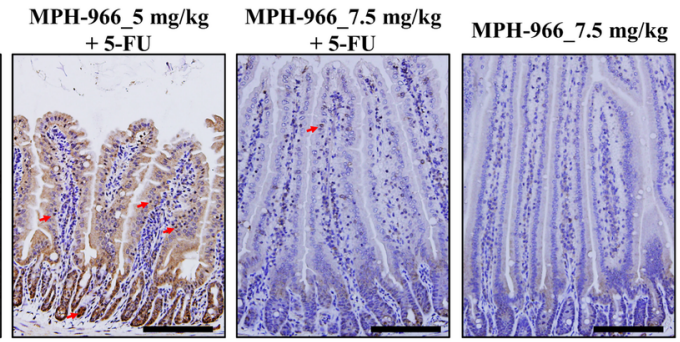
A

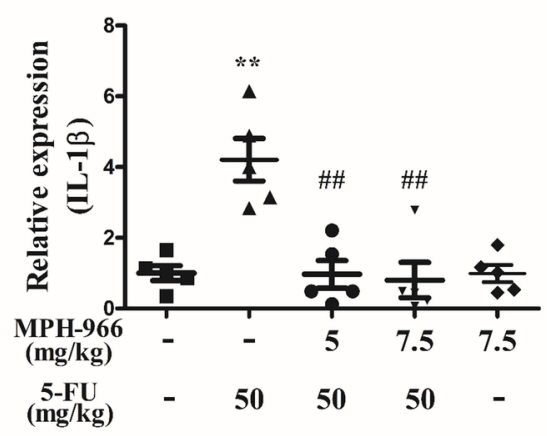

B

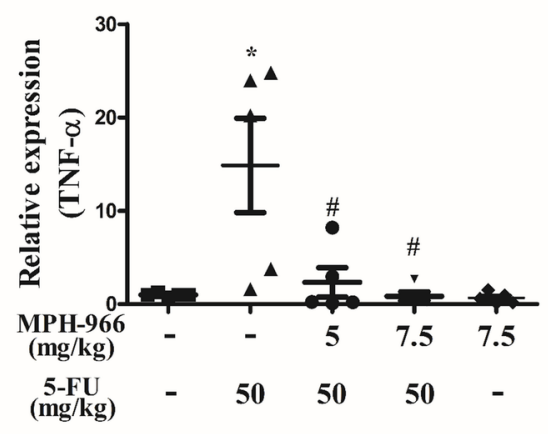

A

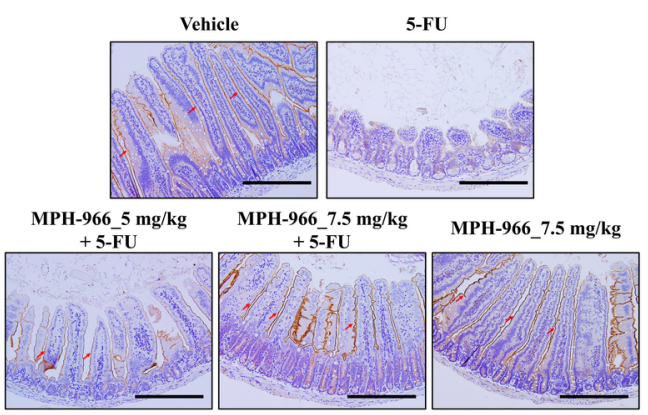

C

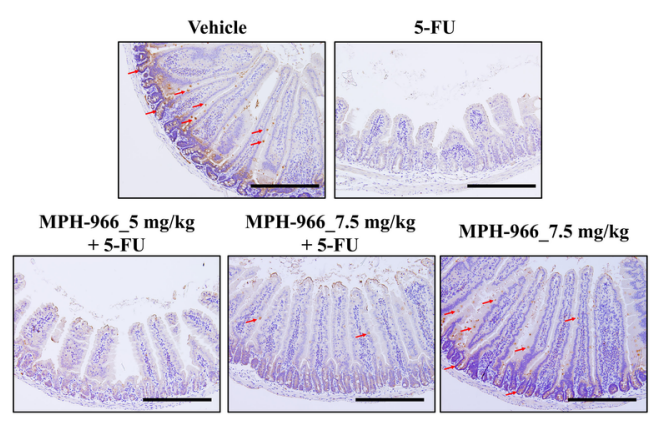

B

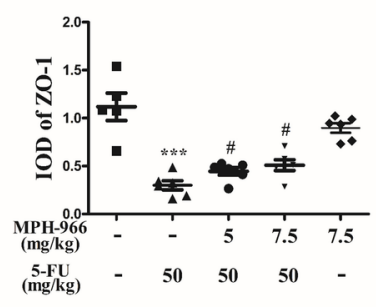

D

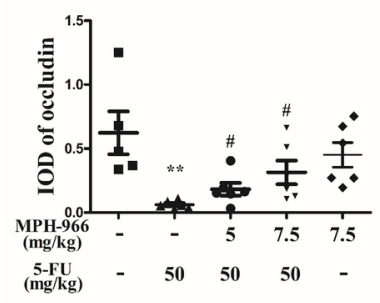




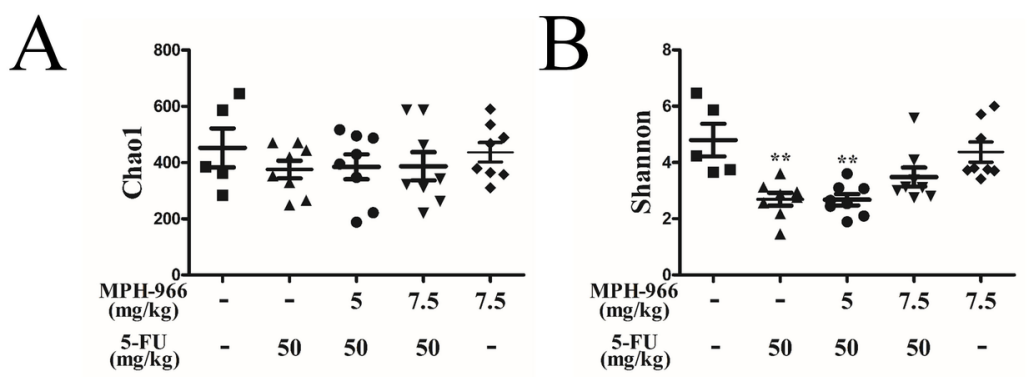

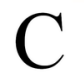
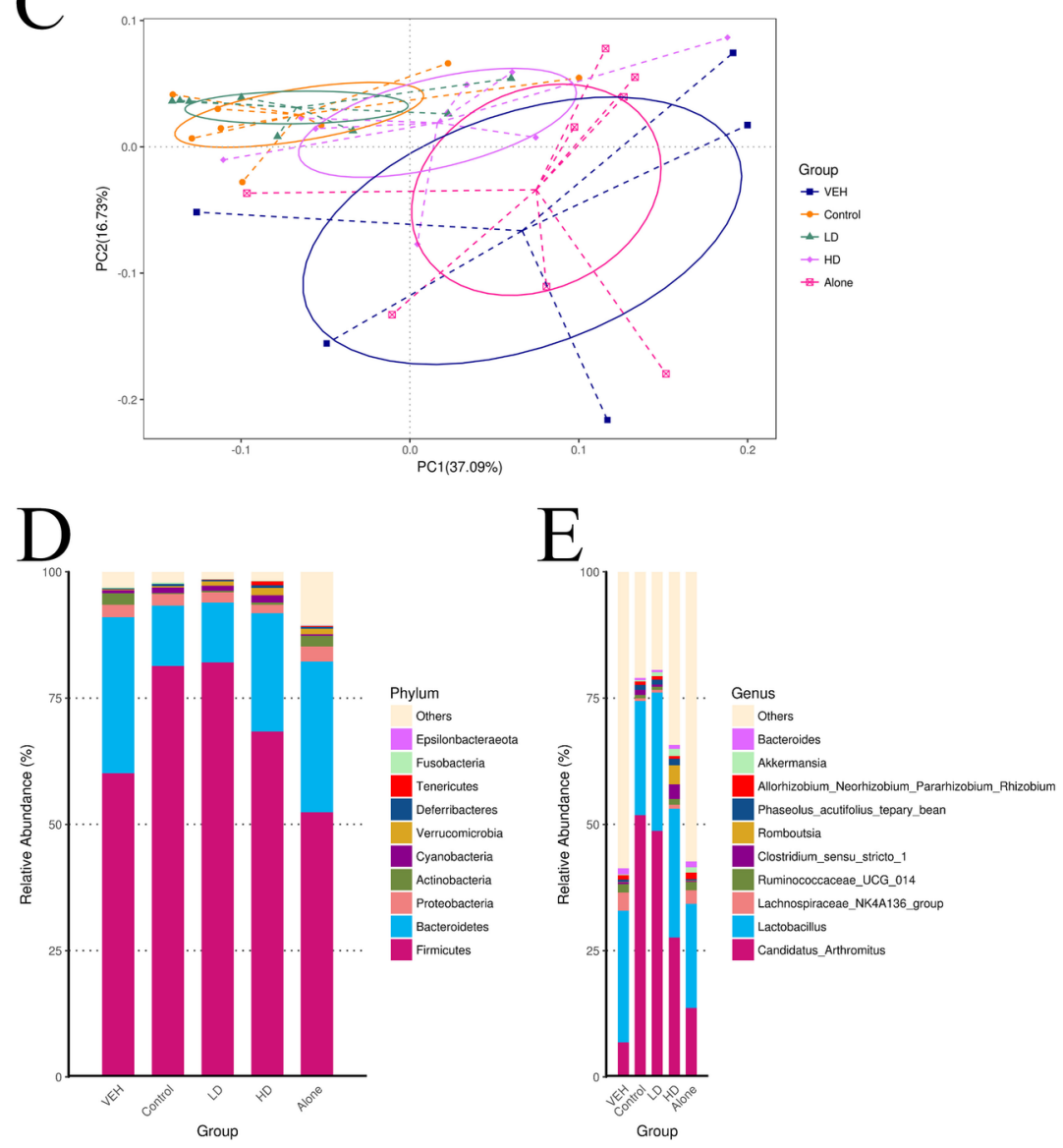


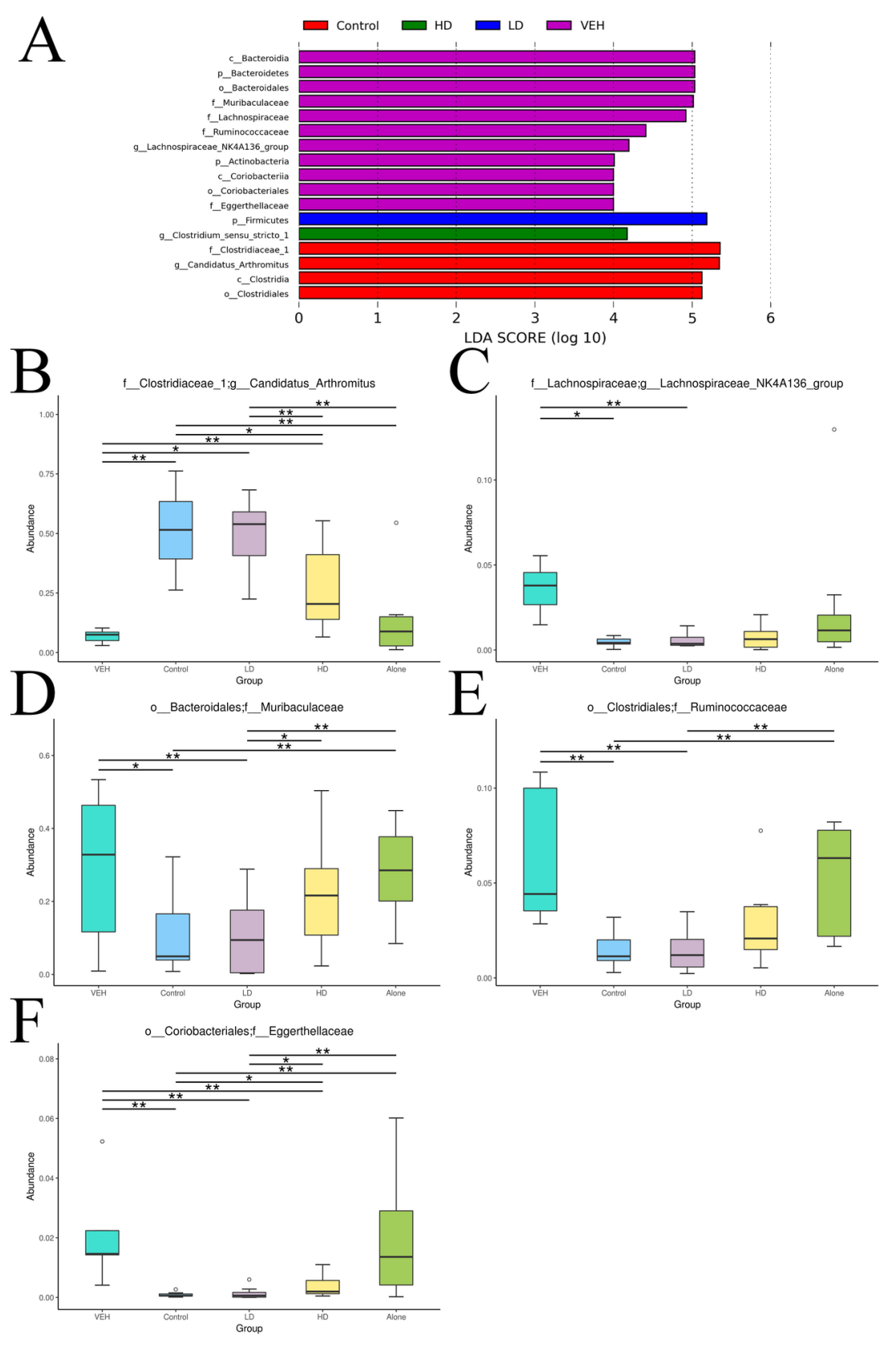


A

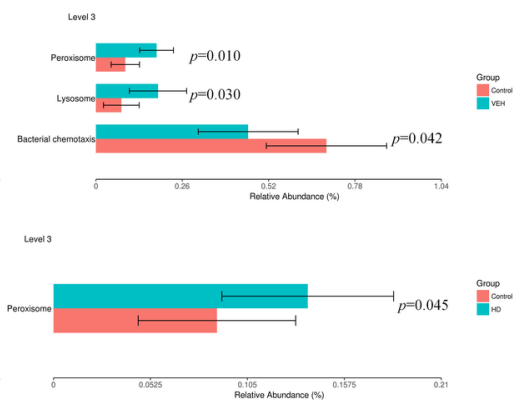

B

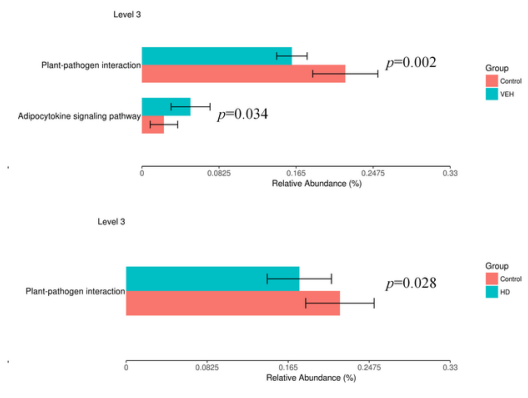


Table 1. Primers sequences

\begin{tabular}{ll} 
Table 1. Primers sequences \\
\hline Gene & Direction and sequence \\
IL-1 $\beta$ & F: 5'-CAGCAGCACATCAACAAGAG-3' \\
TNF- $\alpha$ & R: 5'-GTCACACACCAGCAGGTTAT-3' \\
& F: 5'-TTGCTCTGTGAAGGGAATGG-3' \\
GAPDH & R: 5'-GGCTCTGAGGAGTAGACAATAAAG-3' \\
& F: 5'-AGCTTGTCATCAACGGGAAG-3' \\
& R: 5'-TTTGATGTTAGTGGGGTCTCG-3' \\
\hline
\end{tabular}


Table 2. The relative abundance of top 10 gut microbiota distributed at the phylum level.

\begin{tabular}{lccccc}
\hline $\begin{array}{l}\text { Phyla } \\
\text { Relative abundance (\%) }\end{array}$ & Vehicle & $5-\mathrm{FU}$ & $\begin{array}{c}\mathrm{MPH}-966 \\
\left(\begin{array}{c}5 \mathrm{mg} / \mathrm{kg}) \\
+5-\mathrm{FU}\end{array}\right.\end{array}$ & $\begin{array}{c}\mathrm{MPH}-966 \\
(7.5 \mathrm{mg} / \mathrm{kg}) \\
+5-\mathrm{FU})\end{array}$ & $\begin{array}{c}\mathrm{MPH}-966 \\
(7.5 \mathrm{mg} / \mathrm{kg})\end{array}$ \\
\hline Firmicutes & $60.09 \pm 9.08$ & $81.32 \pm 3.70$ & $82.07 \pm 3.76$ & $68.41 \pm 5.53$ & $52.37 \pm 3.74$ \\
Bacteroidetes & $30.90 \pm 9.92$ & $12.00 \pm 3.92$ & $11.83 \pm 3.83$ & $23.39 \pm 5.41$ & $29.90 \pm 4.39$ \\
Proteobacteria & $2.47 \pm 0.72$ & $2.30 \pm 0.45$ & $2.07 \pm 0.46$ & $1.66 \pm 0.31$ & $2.93 \pm 0.95$ \\
Actinobacteria & $2.28 \pm 0.80$ & $0.17 \pm 0.04$ & $0.27 \pm 0.07$ & $0.47 \pm 0.13$ & $2.13 \pm 0.72$ \\
Cyanobacteria & $0.60 \pm 0.24$ & $1.12 \pm 0.32$ & $1.05 \pm 0.39$ & $1.44 \pm 0.35$ & $0.32 \pm 0.10$ \\
Verrucomicrobia & $0.18 \pm 0.12$ & $0.25 \pm 0.11$ & $0.82 \pm 0.34$ & $1.46 \pm 1.10$ & $1.10 \pm 0.66$ \\
Deferribacteres & $0.17 \pm 0.04$ & $0.43 \pm 0.29$ & $0.31 \pm 0.10$ & $0.56 \pm 0.34$ & $0.34 \pm 0.19$ \\
Tenericutes & $0.03 \pm 0.02$ & $0.05 \pm 0.03$ & $0.04 \pm 0.01$ & $0.70 \pm 0.67$ & $0.17 \pm 0.13$ \\
Fusobacteria & $0.17 \pm 0.09$ & $0.18 \pm 0.09$ & $0.11 \pm 0.05$ & $0.12 \pm 0.04$ & $0.14 \pm 0.09$ \\
Epsilonbacteraeota & $0.04 \pm 0.02$ & $0.03 \pm 0.02$ & $0.03 \pm 0.01$ & $0.02 \pm 0.01$ & $0.07 \pm 0.05$ \\
\hline Firmicutes / Bacteroidetes $(\mathrm{F} / \mathrm{B})$ & 1.94 & 6.78 & 6.94 & 2.93 & 1.75 \\
\hline \multicolumn{7}{l}{ Values were represented as the mean \pm SEM. (n=5 for vehicle group, $\mathrm{n}=8$ for other each groups) }
\end{tabular}

Table 3. The relative abundance of top 10 gut microbiota distributed at the genus level.

\begin{tabular}{lccccc}
\hline $\begin{array}{l}\text { Genera } \\
\text { Relative abundance (\%) }\end{array}$ & Vehicle & $5-\mathrm{FU}$ & $\begin{array}{c}\mathrm{MPH}-966 \\
(5 \mathrm{mg} / \mathrm{kg}) \\
+5-\mathrm{FU}\end{array}$ & $\begin{array}{c}\mathrm{MPH}-966 \\
\left(\begin{array}{c}\mathrm{M} .5 \mathrm{mg} / \mathrm{kg} \\
+5-\mathrm{FU}\end{array}\right.\end{array}$ & $\begin{array}{c}\mathrm{MPH}-966 \\
(7.5 \mathrm{mg} / \mathrm{kg})\end{array}$ \\
\hline Candidatus_Arthromitus & $6.86 \pm 1.30$ & $51.84 \pm 6.52$ & $48.69 \pm 5.72$ & $27.60 \pm 6.77$ & $13.65 \pm 6.25$ \\
Lactobacillus & $26.07 \pm 8.57$ & $22.63 \pm 4.44$ & $27.41 \pm 3.56$ & $25.53 \pm 3.74$ & $20.62 \pm 5.54$ \\
Lachnospiraceae_NK4A136_group & $3.61 \pm 0.71$ & $0.47 \pm 0.10$ & $0.55 \pm 0.14$ & $0.79 \pm 0.28$ & $2.66 \pm 1.51$ \\
Ruminococcacea__UCG_014 & $1.68 \pm 0.66$ & $0.68 \pm 0.25$ & $0.57 \pm 0.21$ & $1.11 \pm 0.34$ & $1.71 \pm 0.46$ \\
Clostridium_sensu_stricto_I & $0.35 \pm 0.04$ & $0.99 \pm 0.25$ & $0.44 \pm 0.09$ & $2.91 \pm 2.43$ & $0.17 \pm 0.04$ \\
Romboutsia & $0.05 \pm 0.03$ & $0.02 \pm 0.01$ & $0.03 \pm 0.01$ & $3.76 \pm 3.60$ & $0.06 \pm 0.04$ \\
Phaseolus_acutifolius_tepary_bean & $0.42 \pm 0.19$ & $0.99 \pm 0.28$ & $0.94 \pm 0.36$ & $1.28 \pm 0.30$ & $0.28 \pm 0.10$ \\
Allorhizobium_Neorhizobium_Pararhizobium_Rhizobium & $0.91 \pm 0.27$ & $0.64 \pm 0.15$ & $0.68 \pm 0.21$ & $0.55 \pm 0.18$ & $1.31 \pm 0.56$ \\
Akkermansia & $0.17 \pm 0.12$ & $0.25 \pm 0.11$ & $0.82 \pm 0.34$ & $1.46 \pm 1.10$ & $1.09 \pm 0.66$ \\
Bacteroides & $1.18 \pm 0.23$ & $0.46 \pm 0.07$ & $0.43 \pm 0.09$ & $0.75 \pm 0.16$ & $1.15 \pm 0.20$ \\
\hline Valles wer_epresnted
\end{tabular}

Values were represented as the mean \pm SEM. ( $\mathrm{n}=5$ for vehicle group, $\mathrm{n}=8$ for other each groups) 